\title{
Detección de Mycoplasma genitalium y correlación con manifestaciones clínicas en una población del estado Zulia, Venezuela
}

\author{
Nailet Arráiz R., Sonia Colina Ch., Rafael Marcucci J., Netxibeth Rondón G., Francia Reyes S., \\ Valmore Bermúdez P. y Zoila Romero F.
}

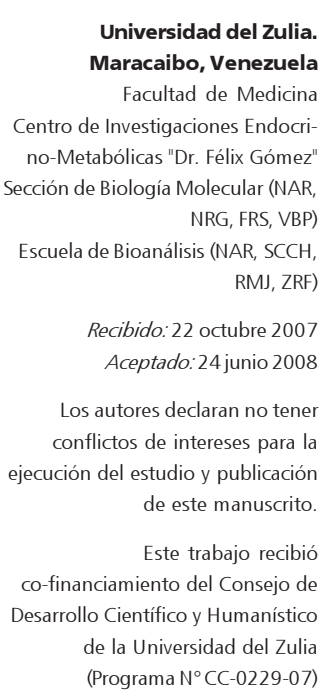

Correspondencia a: Nailet Arráiz Rodríguez narraiz@cantv.net

\section{Mycoplasma genitalium detection and correlation with clinical manifestations in population of the Zulia State, Venezuela}

Diverse studies demonstrate an association between Mycoplasma genitalium and urogenital pathologies. The aim of this study was to investigate the prevalence of $M$. genitalium in patients attending gynecological evaluation in private clinics $(\mathrm{n}=172)$. DNA amplification assays of the genes $16 \mathrm{~S} r R \mathrm{R}$ and $\mathrm{MgPa}$ were utilized. The prevalence of $M$. genitalium in the study population was $7.5 \%$. M. genitalium was detected in $12.1 \%$ and $4.1 \%$ of the symptomatic and asymptomatic patients, respectively $(\mathrm{p}=0.047)$. The infection was diagnosed in patients with cervicitis $(17.2 \%)$ and mucopurulent secretion $(16.6 \%)$ and the highest prevalence of infections was registered in the 31-40 years age group. No significant association between the presence of M.genitalium and individual clinical manifestations or the patients age was showed $(\mathrm{p}>0.05)$. The high prevalence of $M$. genitalium infections, mostly in patients with clinical manifestations showed in this study, warrants the application of diagnostic strategies in the population to investigate the clinical meaning of these microorganisms and to reevaluate therapeutic schemes against non-gonococcal and non-chlamydial infections.

Key words: Mycoplasma genitalium, $16 \mathrm{~S} \mathrm{RNA,MgPa}$, cervicitis, urogenital pathologies, non-gonococcal infections, non-chlamydial infections.

Palabras clave: Mycoplasma genitalium, $16 \mathrm{~S} r A R N, M g P a$, cervicitis, patologías urogenitales, infecciones no gonocóccicas, infecciones no clamidiales.

\section{Introducción}

$\mathrm{L}$ os miembros del género Mycoplasma representan un modelo excepcional que se distingue del resto de los organismos procariotas por poseer un pequeño genoma y carecer de pared celular. El potencial patogénico de estos microorganismos ha sido ampliamente debatido debido a que comúnmente colonizan el tracto genital de hombres y mujeres ${ }^{1-3}$; no obstante, numerosos estudios han reportado una asociación entre Mycoplasma genitalium y patologías urogenitales como uretritis, cervicitis, salpingitis, enfermedad inflamatoria pélvica e infertilidad ${ }^{3-8}$.

Aunque las patologías mencionadas se han asociado clásicamente a infecciones por Chlamydia trachomatis $^{9-12}$ y Neisseria gonorrhoeae $e^{11-13}$, aproximadamente $50 \%$ de los casos se explica por otros agentes causales, entre los cuales debería considerarse la posible contribución de $M$. genitalium.

La principal limitación para la investigación de infecciones por micoplasmas es la dificultad para culti- varlos. La publicación de la secuencia de los genomas de $M$. genitalium $^{14}$ y M. pneumoniae ${ }^{15}$ ha revelado la ausencia de genes de rutas biosintéticas de aminoácidos, lo que hace que estos organismos sean dependientes absolutos de un suministro exógeno, no sólo de aminoácidos, sino de otros precursores biosintéticos como nucleótidos, ácidos grasos y esteroles ${ }^{1}$; en consecuencia, estos microorganismos requieren medios de cultivo y condiciones de crecimiento especia$\operatorname{les}^{16}$, que difícultan el diagnóstico de laboratorio con propósitos de rutina.

Debido a estas limitaciones, en los últimos años se han estandarizado y aplicado técnicas de amplificación de ácidos nucleicos para la investigación de micoplasmas genitales ${ }^{17-20}$ y la mayoría de los estudios clínicos y epidemiológicos en diferentes poblaciones se han realizado utilizando estos ensayos molecula$\operatorname{res}^{5,7,7,18-20}$. Sin embargo, en nuestro medio, estas técnicas no han sido incorporadas a los laboratorios de diagnóstico, por lo cual no existen estudios de la prevalencia de estos microorganismos. 
El objetivo de este trabajo fue investigar la prevalencia de infecciones por $M$. genitalium, a través de ensayos moleculares, en una población de mujeres sexualmente activas que asistieron a consulta de ginecología en centros de salud y correlacionar su presencia con manifestaciones clínicas.

\section{Material y Método}

Se realizó un estudio prospectivo, transversal y descriptivo para evaluar la prevalencia de M. genitalium en una población de mujeres sexualmente activas en zonas urbanas del Municipio de Maracaibo, estado Zulia, Venezuela.

El protocolo de investigación siguió los lineamientos éticos internacionales respetando los acuerdos de la Declaración de Helsinki en su revisión de octubre del año 2000 y fue aprobado por el Consejo de Desarrollo Científico y Humanístico de la Universidad del Zulia. La participación voluntaria de las pacientes en este estudio fue autorizada a través de un documento de consentimiento informado, en el cual se les informó los objetivos de la investigación y se les garantizó confidencialidad y privacidad de los resultados obtenidos.

Selección de la población de estudio. Se evaluaron 172 pacientes del sexo femenino, sexualmente activas, en edades comprendidas entre 20 y 48 años, pertenecientes a clases sociales media alta (Estrato II) y media baja (Estrato III), de acuerdo a la escala de Graffar modificada ${ }^{21}$, que asistieron a consulta de ginecología en tres centros de salud del Municipio de Maracaibo, estado Zulia, Venezuela durante el período agosto 2006 - enero 2007. Se excluyeron pacientes con diagnóstico de infección por Chlamydia trachomatis, virus herpes simplex (VHS-2), virus papiloma humano (VPH) y aquellas que habían recibido terapia antimicrobiana durante las últimas tres semanas. En la población no se detectaron casos de infección por Neisseria gonorrhoeae ni Trichomonas vaginalis.

Durante la evaluación clínica y ginecológica, las pacientes se clasificaron como sintomáticas y asintomáticas. El grupo sintomático incluyó aquellas pacientes que asistieron a consulta de ginecología con manifestaciones clínicas como secreción vaginal anormal, cervicitis, sangrado post-coital, disuria, o cualquier manifestación genito-urinaria. Se registró para cada paciente la manifestación clínica predominante, de acuerdo a criterios del médico especialista. El grupo de pacientes asintomáticas incluyó aquellas que asistieron a consulta ginecológica sin presentar manifestaciones clínicas.
Recolección de la muestra. Previa elaboración de la historia clínica y evaluación ginecológica, a todas las pacientes se les tomó un hisopado de la región endocervical, el cual se transportó en un tubo conteniendo $1 \mathrm{ml}$ de tampón fosfato salino (PBS), $\mathrm{pH} 7,2$. Las muestras se refrigeraron a $-20{ }^{\circ} \mathrm{C}$ y se procesaron durante los tres primeros días de su recepción.

Extracción de $A D N$. Para la extracción de $\mathrm{ADN}$ se utilizó el procedimiento descrito previamente ${ }^{22}$. El hisopo fue presionado enérgicamente contra las paredes del tubo antes de descartarlo y el volumen total de muestra fue transferido a un tubo de $1,5 \mathrm{ml}$ y se centrifugó a 14.000 x g. El sedimento se resuspendió en 500 $\mu l$ de tampón TE (10 mM Tris-HCl, pH 8, 1mM EDTA, $\mathrm{pH} 8)$ y después de este lavado, el sedimento se resuspendió en $400 \mu \mathrm{l}$ de tampón de lisis $(50 \mathrm{mM}$ Tris$\mathrm{Hcl} \mathrm{pH} 7,5,1 \%$ Triton X-100, 1 mMEDTA, $250 \mu \mathrm{g} / \mathrm{ml}$ de proteinasa K). Se incubó la muestra a $56{ }^{\circ} \mathrm{C}$ durante 2 horas. Los lisados fueron extraídos con fenol-cloroformo-alcohol isoamílico $(25: 24: 1)$, precipitados con isopropanol y lavados con etanol $70 \%$. El ADN se resupendió en $30 \mu 1$ de tampón TE. Se utilizó $5 \mu 1$ de la muestra para ensayos de amplificación.

Detección de M. genitalium por reacción de polimerasa en cadena (RPC). Como ensayo preliminar se utilizó un kit un de detección de Maxim Biotech, INC, USA (SP-10522). Este kit incluye oligonucleótidos dirigidos a una secuencia de $\mathrm{ADN}$ conservada del gen $16 S$ rARN (Acceso M24289), que permite amplificar un fragmento de $320 \mathrm{pb}$ del genoma de microorganismos del género Mycoplasma. La mezcla de reacción y condiciones de amplificación por RPC se reprodujeron siguiendo instrucciones del proveedor. El programa de amplificación consistió en una desnaturalización inicial de 8 minutos a $96{ }^{\circ} \mathrm{C}$, seguidos de 35 ciclos de desnaturalización a $94{ }^{\circ} \mathrm{C}$ por un minuto, alineamiento a $58{ }^{\circ} \mathrm{C}$ por 1 minuto y extensión a $72{ }^{\circ} \mathrm{C}$ por 1 minuto. Se hizo una extensión final a $72{ }^{\circ} \mathrm{C}$ durante 10 minutos. Como control positivo, se utilizó el fragmento de ADN clonado en plásmido de la región conservada $16 \mathrm{~S} \mathrm{ARNr}$ que incluye el kit.

Para detectar el genoma de $M$. genitalium en las muestra clínicas se utilizaron secuencias de oligonucleótidos MG1F y MG2R, previamente publicadas ${ }^{17,23}$, que permiten amplificar un fragmento de $282 \mathrm{pb}$ del gen $\mathrm{MgPa}$ (codificante de una adhesina requerida para la fijación de $M$. genitalium a células epiteliales). La mezcla de reacción consistió en $10 \mu 1$ de tampón Go taq $\mathrm{ADN}$ polimerasa 5X (Promega), 1,5 $\mathrm{mM} \mathrm{MgCl}_{2}, 200 \mu \mathrm{M}$ de cada desoxirribonucleótido (dATP, dCTP, dGTP y dTTP), 25 pmoles de cada oligonucleótido, 0,25 $\mu \mathrm{l}$ de Taq ADN polimerasa $5 U / \mu 1$ (Promega) y $5 \mu 1$ de muestra 
de ADN para cada reacción. El programa de amplificación consistió en 8 minutos a $95{ }^{\circ} \mathrm{C}$ y 35 ciclos de amplificación de un minuto a $95^{\circ} \mathrm{C}$, un minuto a $55^{\circ} \mathrm{C}$, un minuto a $72^{\circ} \mathrm{C}$ y un paso final de amplificación a 72 ${ }^{\circ} \mathrm{C}$ por 8 minutos.

Las reacciones de amplificación se llevaron a cabo en un termociclador MJ Research PTC-100 ${ }^{\mathrm{TM}}$. Los productos de RPC se analizaron en geles de agarosa al 1\% y se utilizó tampónr TBE para la corrida electroforética (Tris-Borato $89 \mathrm{mM}$, EDTA $2 \mathrm{mM} \mathrm{pH} \mathrm{8).} \mathrm{La} \mathrm{corrida} \mathrm{se}$ llevó a cabo a $80 \mathrm{v} / \mathrm{cm}$ por una hora. Los geles fueron

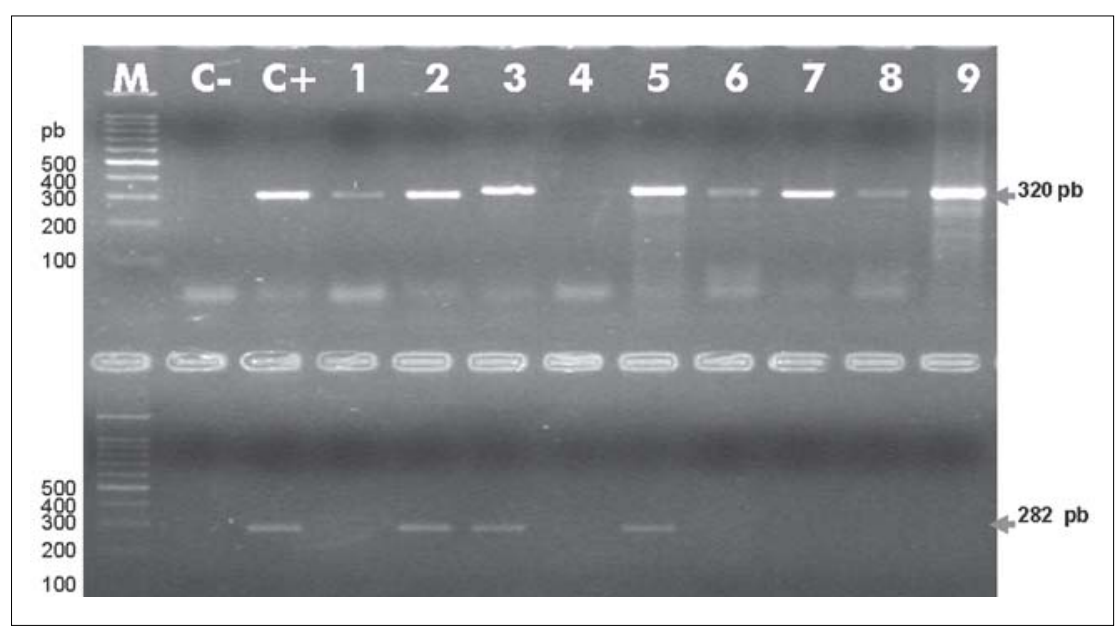

Figura 1. Ensayos de amplificación por RPC de genes 165 rARN (superior) y MgPa (inferior). M: Marcador de peso molecular; C-: control negativo; $C+$ : control positivo; Carriles 1 al 9: muestras de 9 de las pacientes evaluadas, 8 de ellas, positivas para el ensayo 16S rARN (género específico), de las cuales sólo tres muestran el producto específico de $M$. genitalium.

Tabla 1. Prevalencia de infección por Mycoplasma genitalium de acuerdo presencia o no de síntomas y edad de las pacientes

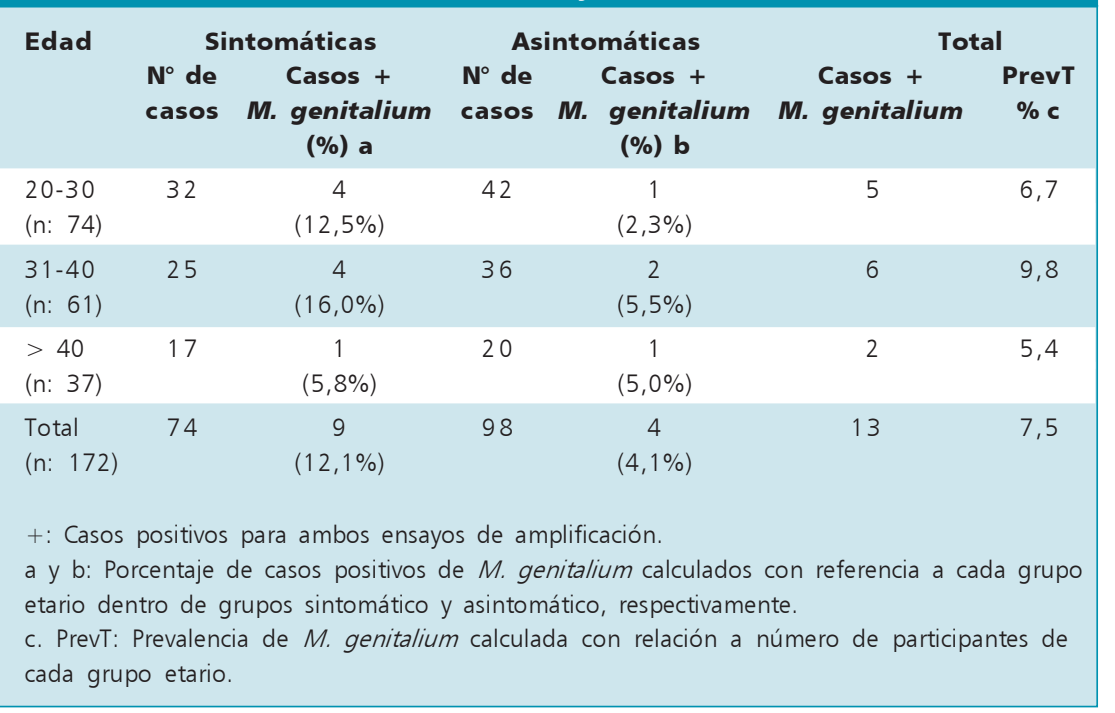

teñidos con bromuro de etidio, visualizados en transiluminador ultravioleta y fotografiados con sistema de fotodocumentación DigiDoc UVP.

Una muestra se consideró positiva cuando se obtuvo amplificación del fragmento de $282 \mathrm{pb}$ en dos reacciones de RPC llevadas a cabo en dos momentos diferentes.

Análisis estadístico. Para el análisis estadístico se utilizó el software SPSS (versión 10) para Windows. A través del análisis descriptivo se calcularon frecuencias con sus respectivos porcentajes para cada una de las variables. La asociación de infección por M. genitalium con manifestaciones clínicas particulares y edad de las pacientes se calculó a través de tablas de contingencia y $\chi^{2}$. Se estableció un nivel de significancia de $\mathrm{p}<0,05$.

\section{Resultados}

Este estudio permitió determinar la prevalencia de infecciones por M. genitalium en 172 mujeres que asistieron a tres centros de salud de zonas urbanas del municipio Maracaibo, estado Zulia, Venezuela. El total de pacientes que asistió a consulta durante el período agosto 2006 - enero 2007 fue de 278. Sólo 209 pacientes aceptaron su participación en el estudio. Durante la evaluación, se excluyeron ocho pacientes con diagnóstico de infección por VPH, 12 pacientes con $C$. trachomatis y 17 pacientes que recibieron terapia antimicrobiana tres semanas previas al estudio. No se detectaron casos de infección por $N$. gonorrhoeae ni T. vaginalis. El promedio de edad de las pacientes fue de 31,4 $\pm 6,5$. De las 172 pacientes, 74 (43\%) exhibieron manifestaciones clínicas y 98 (57\%) fueron asintomáticas al momento de ejecutar el estudio.

En el primer ensayo, se logró amplificar el fragmento esperado de $320 \mathrm{pb}$ del $16 \mathrm{~S}$ rARN en 21 de las 172 muestras $(12,2 \%)$. Estas muestras positivas correspondían a $11 / 74$ pacientes sintomáticas y 10/98 pacientes asintomáticas, resultando en una prevalencia de Mycoplasma sp de 14,8 y 10,2\%, respectivamente.

Al aplicar el segundo ensayo, basado en la amplificación del gen $M g P a$, se definió que sólo 13 de las 21 $(61,9 \%)$ muestras positivas para el ensayo géneroespecífico, contenían el genoma de $M$. genitalium. En la Figura 1 se ilustra el patrón de amplificación de nueve muestras, ocho de las cuales fueron positivas para el ensayo $16 S$ rARN y sólo tres resultaron positivas para $M$. genitalium.

Este análisis permitió estimar una prevalencia de $M$. genitalium en la población de 7,5\% (Tabla 1). En este estudio no se llevó a cabo la confirmación de la identi- 
dad de especies de Mycoplasma detectados en las ocho muestras restantes $(38,1 \%)$, positivas para el ensayo $16 S r A R N$. Estos casos se ubicaron dentro del grupo de pacientes asintomáticas.

Mycoplasma genitalium fue detectado en nueve de las 74 pacientes que exhibieron manifestaciones clínicas $(12,1 \%)$ y cuatro de las 98 pacientes asintomáticas $(4,1 \%)$ (Tabla 1), evidenciándose una diferencia significativa entre ambos grupos (p: 0,047). La mayor prevalencia de infecciones por $M$. genitalium se registró en el grupo etario de 31-40 años, pero no se encontró diferencia significativa al correlacionar la presencia de $M$. genitalium con la edad de las pacientes $(\mathrm{p}>0,05)$.

Entre las manifestaciones clínicas registradas con mayor frecuencia en esta población se destacaron cervicitis $(39,2 \%)$, disuria $(27,0 \%)$ y secreción mucopurulenta (16,2\%) (Tabla 2). El mayor número de casos de M. genitalium fue detectado en pacientes con cervicitis $(17,2 \%)$ y con secreción mucopurulenta $(16,6 \%)$; sin embargo, no se encontró asociación significativa de infección por M. genitalium con alguna manifestación clínica en particular.

\section{Discusión}

Las dificultades para el diagnóstico de infecciones por M. genitalium, ha limitado el conocimiento de la epidemiología de este microorganismo en nuestro medio, por lo cual se ha realizado el presente estudio, utilizando una estrategia de diagnóstico molecular que podría ser de gran valor como herramienta para la investigación de infecciones por $M$. genitalium y permitiría evaluar la prevalencia de este patógeno en poblaciones a gran escala.

La prevalencia de infecciones por $M$. genitalium encontrada en este estudio es equivalente a la reportada por varios autores, ubicándose en el rango de 1 a $10 \%$ en la mayoría de las poblaciones ${ }^{24-27}$. Mycoplasma genitalium es detectado con mayor frecuencia en poblaciones con factores de riesgo ${ }^{28}$, alcanzando cifras de 20 a $30 \%$ en trabajadoras sexuales ${ }^{29}$.

La prevalencia de 7,5\% resultó superior a los resultados esperados para esta población perteneciente a clases sociales media alta y media baja ${ }^{21}$, que incluye mujeres con características cognitivo-perceptuales en las cuales se presume auto-eficacia para asumir conductas preventivas y favorecedoras con respecto a su salud y con condiciones socio-económicas que le permiten acceso permanente a los servicios de salud reproductiva. Lamentablemente no se pudo establecer asociación de la infección con factores de riesgo, debido a que sólo un número reducido de pacientes acce-
Tabla 2. Mycoplasma genitalium y manifestaciones clínicas individuales

\begin{tabular}{|c|c|c|c|c|c|}
\hline \multirow[b]{2}{*}{ Signos y síntomas } & \multicolumn{2}{|c|}{ Pacientes } & \multicolumn{3}{|c|}{$\begin{array}{c}\text { Casos + Mycoplasma } \\
\text { genitalium }\end{array}$} \\
\hline & $\mathbf{n}$ & $\%$ & $n$ & $\%^{a}$ & $\%^{b}$ \\
\hline Cervicitis & 29 & 39,2 & 5 & 17,2 & 6,7 \\
\hline Secreción mucopurulenta & 12 & 16,2 & 2 & 16,6 & 2,7 \\
\hline Disuria & 20 & 27,0 & 1 & 5,0 & 1,3 \\
\hline Sangrado post-coital & 9 & 12,1 & 0 & 0 & 0 \\
\hline Enfermedad inflamatoria pélvica & 4 & 5,4 & 1 & 25,0 & 1,3 \\
\hline Total & 74 & 100 & 9 & 12,1 & 12,1 \\
\hline
\end{tabular}

dió a llenar un cuestionario exploratorio para evaluar de manera sistemática y objetiva aspectos relacionados con sus hábitos sexuales. Algunos autores señalan que el reporte voluntario del comportamiento sexual se ha convertido en un pobre predictor del riesgo de infección, al no garantizar la fidelidad de la información suministrada, motivado principalmente por la estigmatización de las infecciones de transmisión sexual ${ }^{30}$.

Un hallazgo relevante en este trabajo fue la ausencia de asociación de $M$. genitalium con signos y síntomas particulares; sin embargo, la asociación significativa encontrada entre $M$. genitalium y la presencia de manifestaciones clínicas en general, representa nuevas evidencias que apoya la hipótesis sobre la potencial contribución de $M$. genitalium a morbilidades del tracto urogenital ${ }^{3-8,25,27}$

En un trabajo reciente, estos síntomas fueron asociados fuertemente con especies de Ureaplasma, acompañado de una baja prevalencia de $M$. genitalium; sin embargo, en ese estudio, los autores reportaron que la condición subóptima de amplificación para este microorganismo podría ser responsable de su baja frecuencia de detección ${ }^{31}$

M. genitalium parece localizarse en el extra e intracelular en presencia de una infección moderada y predominantemente en el intracelular en los casos de infección grave, sugiriendo una invasión masiva de Mycoplasma acompañado de replicación intracelular y persistencia ${ }^{32,33}$. De acuerdo a este patrón de infección, sería recomendable detectar tempranamente la infección y tratarla en estadios tempranos, debido a que la persistencia intracelular podría dificultar el éxito de la terapia antimicrobiana.

La mayoría de mujeres con sintomatología urogenital son tratadas empíricamente contra $N$. gonorrhoeae y C. trachomatis, de acuerdo a lineamientos para el tra- 
tamiento de enfermedades de transmisión sexual ${ }^{12}$; sin embargo, deberían ser evaluados esquemas terapéuticos, que consideren las evidencias acumuladas hasta el momento a través de múltiples reportes, incluyendo los resultados de este trabajo, que sugieren una potencial contribución de $M$. genitalium al desarrollo de patologías del tracto urogenital no gonocóccicas. Se debería tomar en cuenta que algunos antimicrobianos prescritos, tales como penicilinas y cefalosporinas, actúan a nivel de la pared celular, siendo inefectivos contra $M$. genitalium.

En conclusión, M. genitalium debería ser considerado un potencial patógeno del tracto urogenital cuando es detectado en ausencia de $N$. gonorrhoeae y $C$. trachomatis. Dada la alta prevalencia y asociación encontrada entre $M$. genitalium y manifestaciones clínicas urogenitales en este reporte y otros trabajos, es recomendable aplicar estrategias diagnósticas para investigar el significado clínico de estos microorganismos y reevaluar esquemas terapéuticos contra infecciones no gonocóccicas y no clamidiales.

Agradecimientos. A las pacientes y médicos especialistas que participaron en el estudio. Al Consejo de Desarrollo Científico y Humanístico de la Universidad del Zulia por el cofinanciamiento de esta investigación (Programa $\mathrm{N}^{\circ} \mathrm{CC}$ 0229-07). A la Oficina de Planificación del Sector Universitario (OPSU) por su contribución en el fortalecimiento del laboratorio de Biología Molecular del Centro de Investiga- ciones Endocrino-Metabólicas Dr. Félix Gómez de la Facultad de Medicina de la Universidad del Zulia.

\section{Resumen}

Diversos estudios demuestran una asociación entre Mycoplasma genitalium y patologías urogenitales. El objetivo de este trabajo fue investigar la prevalencia de infecciones por $M$. genitalium en pacientes atendidas en clínicas privadas $(\mathrm{n}=172)$. Se utilizaron ensayos de amplificación de genes $16 S \mathrm{rARN}$ y $\mathrm{MgPa}$. La prevalencia de $M$. genitalium en esta población fue 7,5\%. Mycoplasma genitalium fue detectado en 12,1 y $4,1 \%$ de las pacientes sintomáticas y asintomáticas, respectivamente $(\mathrm{p}=0,047)$. La infección se diagnosticó en pacientes con cervicitis $(17,2 \%)$ y con secreción mucopurulenta $(16,6 \%)$ y la mayor prevalencia de infecciones se registró en el grupo etario de 31 a 40 años. No se encontró asociación significativa entre la presencia de $M$. genitalium y manifestaciones clínicas individuales o edad de las pacientes $(p>0,05)$. La alta prevalencia de infecciones por $M$. genitalium, principalmente en pacientes con manifestaciones clínicas demostrada en este estudio, demanda la aplicación de estrategias diagnósticas en la población para investigar el significado clínico de estos microorganismos y reevaluar esquemas terapéuticos contra infecciones no gonocóccicas y no clamidiales.

\section{Referencias}

1.- Razin S, Yogev D, Naot Y. Molecular biology and pathogenicity of Mycoplasmas. Microbiol Mol Biol Rev 1998; 62: 1094156.

2.- Taylor-Robinson D. Infections due to species of Mycoplasma and Ureaplasma: an update. Clin Infect Dis 1996; 23: 671-84.

3.- Horner P, Thomas B, Gilroy C B, Egger M, Taylor-Robinson D. Role of Mycoplasma genitalium and Ureaplasma urealyticum in acute and chronic nonogonococcal urethritis. Clin Infect Dis 2001; 32: $995-$ 1003 .

4.- Pepin J, Sobela F, Desandes S, Alary M, Wegner K, Khonde N, et al. Etiology of urethral discharge in West Africa: the role of Mycoplasma genitalium and Trichomonas vaginalis. Bull. WHO 2001; 79: 118-26.

5.- Falk J, Fredlund H, Hensen J S. Signs and symptoms of urethritis and cervicitis among women with or without Mycoplasma genitalium or Chlamydia trachomatis infection. Sex Transm Infect 2005; 81: 73-8.
6.- Schwartz M A, Hooton T M. Etiology of non-gonococcal non-chlamydial urethritis. Dematol Clin 1998; 16: 727-33.

7.- Haggerty C I, Hillier S L, Bass D C, Ness R B. Bacterial vaginosis and anaerobic bacteria are associated with endometritis. Clin Infect Dis 2004; 39: 990-95.

8.- Haggerty C I, Totten P A, Astete S G, Ness R B. Mycoplasma genitalium among women with non-gonococcal, nonchlamydial pelvic inflammatory disease. Infect Dis Obstet Gynecol 2006; 2006: $1-5$.

9.- Cates W Jr, Wasserheit J N. Genital chlamydial infections: epidemiology and reproductive sequelae. Am J Obstet Gynecol 1991; 164: 1771-81.

10.- Roberts T E, Robinson S, Barton P M, Bryan S, McCarthy A, Macleod J, et al. Cost effectiveness of home based population screening for Chlamydia trachomatis in the UK: economic evaluation of Chlamydia screening studies (ClaSS) project. Br Med J 2007; 335: 291-97.

11.- Cates W, Rolfs R T, Aral S O. Sexually transmitted diseases, pelvic inflamation diseases and infertility an epidemiologic update. Epidemiol Rev 1990; 19 : 199-220

12.- Centers for Disease Control and Prevention. Sexually transmitted diseases treatment guidelines. MMWR Recomm Rep 2006; 55: 1-94. Erratum in MMWR Recomm Rep 2006; 15: 55: 997.

13.- Monif G R, Welkos S L, Baer H, Thompson R J. Cul-de-sac isolates from patients with endometritis-salpingitisperitonitis and gonococcal endocervicitis. American J Obstetr Ginecol 1976; 126: 158-61

14.- Fraser C M, Gocayne J D, White O, Adams M D, Clayton R A, Fleiscmann R D, et al. The minimal gene complement of Mycoplasma genitalium. Science 1995; 270 : 397-403.

15.- Himmelreich R, Hilbert $H$, Plagens $H$, Pirkl E, Li B-C, Herrmann R. Complete sequence analysis of the genome of the bacterium Mycoplasma pneumoniae. Nucleic Acids Res 1996; 24: 4420-49.

16.- Hamasuna R, Osada Y, Jensen JS. Isolation of Mycoplasma genitalium from first-void 
urine specimens by co-culture with Vero Cells. J Clin Microbiol 2007; 45: 847-50

17.- Stellrecht K A, Woron A M, Mishrik G, Venezia R A. Comparison of Multiplex PCR assay with culture for detection of genital Mycoplasmas. J Clin Microbiol 2004; 42: 1528-33.

18.- Jensen J S, Borre M B, Dohn B. Detection of Mycoplasma genitalium by PCR amplification of the 16S rRNA gene. J Clin Microbiol 2003; 41: 261-6.

19. - Baseman J B, Cagle M, Korte J, Herrera C, Rasmussen W G, Baseman J G, et al. Diagnostic assessment of Mycoplasma genitalium in culture-positive women. J Clin Microbiol 2004; 42: 203-11.

20.- Totten P A, Schwartz K E, Sjostrom K E, Kenny G E, Handsfield H H, Weiss J B, et al Association of Mycoplasma genitalium with nongonococcal urethritis in heterosexual men. J Infect Dis 2001; 183: 269-76.

21.- Méndez-Castellano H, De Méndez M C. Estratificación social y biología humana: método de Graffar modificado. Arch Ven Pueric Pediatr 1986; 49: 93-104.

22.- Arráiz N, Ginestre M, Perozo A, Castellano M, Urdaneta B, García M. Diagnóstico molecular y prevalencia de infecciones por Chlamydia trachomatis en pacientes sintomáticas y asintomáticas de una población del estado Zulia, Venezuela.
Rev Chil Infect 2007; 24: 48-52.

23.- Skov-Jensen J, Uldum S A, SondergardAndersen J, Vuust J, Lind K. Polymerase chain reaction for the detection of Mycoplasma genitalium in clinical samples. J Clin Microbiol 1991; 29: 46-50.

24.- Manhart L E, Holmes K K, Hughes J P, Houston L S, Totten P A. Mycoplasma genitalium among young adults in the United Satates: An emerging sexually transmitted infection. Am J Public Health 2007; 97: 118-25.

25.- Anagrius C, Loré B, Jenen J S. Mycoplasma genitalium: prevalence, clinical significance and transmisión. Sex Transm Infect 2005; 81: 458-62.

26.- Andersen B, Sokolowski I, Østergaard L, Møller K, Olesen F, Jensen J S. Mycoplasma genitalium: prevalence and behavioral risk factors in the general population. Sex Transm Infect 2007; 83: 237-41.

27. - Manhart L E, Crithlow C W, Holmes K K, Dutro S M, Eschenbach D A, Stevens C E, et al. Mucopurulent cervicitis and Mycoplasma genitalium. J Infect Dis 2003; 187: 650-7.

28. - Tosh A K, Van Der Pol B, Fortenberry J D, Williams J A, Katz B P, Batteiger B E, et al. Mycoplasma genitalium among adolescent women and their partners. J Adolesc Health 2007; 40: 412-7.
29.- Cohen C R, Nosek M, Meier A, Astete S G, Iverson-Cabral S, Mugo N R, et al. Mycoplasma genitalium infection and persistence in a cohort of female sex workers in Nairobi, Kenia. Sex Transm Dis 2007; 34: 274-9.

30.- Gutiérrez J P, Bertozzi S M, Conde-Glez C J, Sánchez-Alemán M A. Risk behaviour of 15-21 years old in Mexico lead to a high prevalence of sexually transmitted infections: results of a survey in disadvantaged urban areas. BMC Public Health 2006; 6:49-59.

31.- Schlicht M J, Lovrich S D, Sartin J S, Karpinsky P, Cllister S M, Agger W A. High prevalence of genital Mycoplasmas among sexually active young adults with urethritis or cervicitis symptoms in La Crosse, Wisconsin. J Clin Microbiol 2004; 42: 463640

32.- Blaylock M W, Musatovova O, Baseman J G, Baseman J B. Determination of infectious load of Mycoplasma genitalium in clinical samples of human vaginal cells. J Clin Microbiol 2004; 42: 746-52.

33. - Dallo S F, Baseman J B. Intracellular DNA replication and long-term survival of pathogenic mycoplasmas. Microb Pathog 2000; 29: 301-9. 